\title{
On Quantum Differential Subordination Related with Certain Family of Analytic Functions
}

\author{
Afis Saliu $\mathbb{D}^{1},{ }^{1}$ Khalida Inayat Noor, ${ }^{1}$ Saqib Hussain $\mathbb{D D}^{2},{ }^{2}$ and Maslina Darus ${ }^{3}$ \\ ${ }^{1}$ Department of Mathematics, COMSATS University Islamabad, Park Road, Tarlai Kalan, Islamabad 45550, Pakistan \\ ${ }^{2}$ Department of Mathematics, COMSATS University Islamabad, Abbottabad Campus 22060, Abbottabad, Pakistan \\ ${ }^{3}$ Department of Mathematical Sciences, Universiti Kebangsaan Malaysia, Bangi 43600, Selangor, Malaysia
}

Correspondence should be addressed to Afis Saliu; saliugsu@gmail.com

Received 9 October 2020; Revised 16 October 2020; Accepted 21 October 2020; Published 10 November 2020

Academic Editor: Hijaz Ahmad

Copyright (C) 2020 Afis Saliu et al. This is an open access article distributed under the Creative Commons Attribution License, which permits unrestricted use, distribution, and reproduction in any medium, provided the original work is properly cited.

Recently, there is a rapid increase of research in the area of Quantum calculus (known as $q$-calculus) due to its widespread applications in many areas of study, such as geometric functions theory. To this end, using the concept of $q$-conic domains of Janowski type as well as $q$ - calculus, new subclasses of analytic functions are introduced. This family of functions extends the notion of $\alpha$-convex and quasi-convex functions. Furthermore, a coefficient inequality, sufficiency criteria, and covering results for these novel classes are derived. Besides, some remarkable consequences of our investigation are highlighted.

\section{Introduction}

Recently, there is a rapid increase in the area of Quantum calculus (known as $q$-calculus) due to its widespread applications in many areas of study such as geometry functions theory (GFT), combinatorial analysis, Lie theory, mechanical engineering, cosmology, and statistics. The concept of $q$-integral was first introduced and studied by Jackson et al. [6] at the beginning of the twentieth century.

The development of the concept of $q$-calculus in GFT had its history from the work of Ismail et al. [5], where the notion of $q$-starlike functions was extensively studied. As such, many subclasses of univalent functions correlated with $q$-calculus have been on increase (see $[1,14,17,20,23-25,27,28])$. In recent times, various family of $q$ - extension of starlike functions, which are connected to Janowski functions in the open unit disc $U$, were initiated and examined from many different viewpoints and perspective (see $[17,20,28]$ ).

In an attempt to generalize the notion of uniformly closed-to-convex functions considered by Goodman [3], Kanas and Wisniowska $[8-10,13]$ and Kanas and Srivastava [12] introduced the conic domain $\Omega_{m}(m \geq 0)$ and studied the classes $m$ - UCV and $m$ - UST of $m$-uniformly convex and starlike functions. Furthermore, Noor and Malik [22], using the concept of Janowski class, extended the domain $\Omega_{m}$ to $\Omega_{m}(\gamma, \lambda),-1 \leq \lambda<\gamma \leq 1$. In the latest article by Mahmood et al. [17], the importance of $q$-calculus was used to improve the Noor-Malik conic domains to $\Omega_{q, m}(\gamma, \lambda)$. Using this domain, they examined the coefficient inequalities associated with the class $m$ - $\operatorname{UST}_{q}(\gamma, \lambda)$ of $q$-uniformly starlike functions. Afterward, the same coefficient problems were also explored for the classes $m-\mathrm{UCV}_{q}(\gamma, \lambda), m-\mathrm{UKV}_{q}(\gamma, \lambda), m-\mathrm{UC}^{*} V_{q}(\gamma, \lambda) \quad$ of $m$-uniformly $q$-convex, close-to-convex and quasi-convex functions by Naeem et al. [20].

Motivated by these recent articles [15, 17, 20, 28], our aim is to introduce the novel classes $m-\operatorname{UM}_{q}(\alpha, \gamma, \lambda)$ and $m-\mathrm{UQ}_{q}(\alpha, \gamma, \lambda)$ consisting of $m$-uniformly $q$-alpha convex and quasi-convex functions. We study the coefficient inequalities associated with these classes and some other related properties. Some relevant consequences of our results which were studied in previous work show the significance of our investigation.

\section{Materials and Methods}

Now, we give some useful preliminaries which are necessary for our study. 
Let $\mathscr{A}$ be the class of normalized analytic functions $f(\varsigma)$ in $U=\{\varsigma:|\varsigma|<1\}$ with

$$
f(\varsigma)=\varsigma+a_{2} \varsigma^{2}+a_{3} \varsigma^{3}+\cdots
$$

Let $S, \mathrm{CV}, \mathrm{ST}, \mathrm{QV}$, and $\mathrm{KV}$ be the subclasses of $\mathscr{A}$ consisting functions that are univalent, convex, starlike, quasi-convex, and close-to-convex functions, respectively. The function $f(\varsigma)$ of form (1) is subordinate to the analytic function $g(\varsigma)$ (written as $f(\varsigma)<g(\varsigma)$ ) of the form

$$
g(\varsigma)=\varsigma+b_{2} \varsigma^{2}+b_{3} \varsigma^{3}+\cdots,
$$

if there exists a Schwarz function $w(\varsigma)$ in $U$ such that

$$
f(\varsigma)=g(w(\varsigma)), \quad \varsigma \in U
$$

Let $-1 \leq \lambda<\gamma \leq 1$. Then, class $P(\gamma, \lambda)$ (see [7]) of function $p(\varsigma)$ satisfies the subordination condition

$$
p(\varsigma) \prec \frac{1+\gamma \varsigma}{1+\lambda \varsigma}, \quad \varsigma \in U
$$

or equivalently,

$$
p(\varsigma)=\frac{(1+\gamma) h(\varsigma)+(1-\gamma)}{(1+\lambda) h(\varsigma)+(1-\lambda)}, \quad \varsigma \in U,
$$

where $h \in P$ (class of functions with positive real part). For $\gamma=1-2 \beta, 0 \leq \beta<1$, and $\lambda=-1$, the class $P(\gamma, \lambda)$ reduces to the class $P(\beta)$, the class of functions whose real part is greater than $\beta$.

The conic domains $\Omega_{m}(\gamma, \lambda)(m \geq 0)$ of Janowski type introduced by Noor and Malik [22] are defined as follows:

$$
\begin{aligned}
& \Omega_{m}(\gamma, \lambda)=\left\{u+i v:\left[\left(\lambda^{2}-1\right)\left(u^{2}+v^{2}\right)-2(\gamma \lambda-1) u+\left(\gamma^{2}-1\right)\right]^{2}\right. \\
& \left.>m^{2}\left[\left(-2(\lambda+1)\left(u^{2}+v^{2}\right)+2(\gamma+\lambda+2) u-2(\gamma+1)\right)^{2}+4(\gamma-\lambda)^{2} v^{2}\right]\right\} . \\
& \text { rpretation of } \Omega_{m}(\gamma, \lambda) \text { and its effect on } \quad p(\varsigma)<\frac{(\gamma+1) p_{m}(\varsigma)-(\gamma-1)}{(\lambda+1) p_{m}(\varsigma)-(\lambda-1)}, \quad m \geq 0,-1 \leq \lambda<\gamma \leq 1,
\end{aligned}
$$

Geometrical interpretation of $\Omega_{m}(\gamma, \lambda)$ and its effect on $\Omega_{m}$ were also demonstrated in [22]. The class $m-P(\gamma, \lambda)$ represents the class of all functions that maps $U$ onto $\Omega_{m}(\gamma, \lambda)$. Equivalently, a function $p(\varsigma)$ belongs to

where $p_{m}(\varsigma)$ has its definition in $[10,11]$ and given by $m-P(\gamma, \lambda)$ if and only if

$$
p_{m}(\varsigma)=\left\{\begin{array}{l}
\frac{1+\varsigma}{1-\varsigma}, \quad m=0, \\
1+\frac{2}{\pi^{2}}\left(\log \frac{1+\sqrt{\varsigma}}{1-\sqrt{\varsigma}}\right)^{2}, \quad m=1, \\
1+\frac{2}{1-m^{2}} \sinh ^{2}\left[\left(\frac{2}{\pi} \arccos m\right) \arctan \sqrt{\varsigma}\right], \quad 0<m<1, \\
1+\frac{1}{1-m^{2}} \sin \left[\frac{\pi}{2 R(t)} \int_{0}^{u(\varsigma) / \sqrt{t}} \frac{1}{\sqrt{1-x^{2}} \sqrt{1-(t x)^{2}}} \mathrm{~d} x\right]+\frac{1}{m^{2}-1}, \quad m>1,
\end{array}\right.
$$

where $u(\varsigma)=((\varsigma-\sqrt{t}) /(1-\sqrt{t \varsigma})), t \in(0,1), \varsigma \in U$ and $t$ is chosen such that $m=\cosh \left(\left(\pi R^{\prime}(t)\right) /(4 R(t))\right), R(t)$ is Legendre's complete elliptic integral of the first kind, and $R^{\prime}(t)$ is the complementary integral of $R(t)$
Definition 1 (see [2]). Let $q \in(0,1)$. Then, the $q$-number $[n]_{q}$ is given as

$$
[n]_{q}=\left\{\begin{array}{l}
\frac{1-q^{n}}{1-q}, \quad n \in \mathbb{C}, \\
\sum_{l=0}^{n-1} q^{l}=1+q+q^{2}+\cdots+q^{n-1}, \quad n \in \mathbb{N}, n, \quad \text { as } q \longrightarrow 1^{-},
\end{array}\right.
$$


and the $q$-derivative of a complex valued function $f(\varsigma)$ in $U$ is given by

$$
D_{q} f(\varsigma)=\left\{\begin{array}{l}
\frac{f(q \varsigma)-f(\varsigma)}{(q-1) \varsigma}, \quad \varsigma \neq 0, \\
f^{\prime}(0), \quad \varsigma=0, \\
f^{\prime}(\varsigma), \quad \text { as } q \longrightarrow 1^{-} .
\end{array}\right.
$$

From the above explanation, it is easy to see that, for $f(\varsigma)$ given by (1),

$$
D_{q} f(\varsigma)=1+\sum_{l=2}^{\infty}[\iota]_{q} a_{\iota} \varsigma^{l}
$$

Definition 2 (see [28]). An analytic function $p(\varsigma)$ in $U$ belongs to $P_{q}(\gamma, \lambda)$ if and only if the condition

$$
p(\varsigma)=\frac{\left(O_{1} \gamma+O_{3}\right) h(\varsigma)-(\gamma-1) O_{1}}{\left(O_{1} \lambda+O_{3}\right) h(\varsigma)-(\lambda-1) O_{1}},
$$

is satisfied, where $O_{1}=1+q, O_{3}=3-q$ and $h \in P$.

Definition 3 (see [17]). An analytic function $p(\varsigma)$ in $U$ belongs to $m-P_{q}(\gamma, \lambda)$ if and only if the subordination condition

$$
p(\varsigma) \prec \frac{\left(O_{1} \gamma+O_{3}\right) p_{m}(\varsigma)-(\gamma-1) O_{1}}{\left(O_{1} \lambda+O_{3}\right) p_{m}(\varsigma)-(\lambda-1) O_{1}},
$$

is satisfied, where $p_{m}(\varsigma)$ is given by (8). Equivalently, $p \in m-P_{q}(\gamma, \lambda)$ if and only if $p(\varsigma)$ conformally maps $U$ onto the domain $\Omega_{q, m}(\gamma, \lambda)$ defined by

$$
\Omega_{q, m}(\gamma, \lambda)=\{p(\varsigma)=u+i v: \operatorname{Re}(\Phi)>m|\Phi-1|\},
$$

where

$$
\Phi=\frac{(\lambda-1) O_{1} p(\varsigma)-(\gamma-1) O_{1}}{\left(\lambda O_{1}+O_{3}\right) p(\varsigma)-\left(\gamma O_{1}+O_{3}\right)} .
$$

Equivalently,

$$
\begin{aligned}
\Omega_{q, m}(\gamma, \lambda)= & \left\{u+i v: O_{1}^{2}\left[(\lambda-1)\left(\lambda O_{1}+O_{3}\right)\left(u^{2}+v^{2}\right)-\left((\lambda-1)\left(\gamma O_{1}+O_{3}\right)\right.\right.\right. \\
& \left.\left.+(\gamma-1)\left(\lambda O_{1}+O_{3}\right) u+(\gamma-1)\left(\gamma O_{1}+O_{3}\right)\right)\right]^{2} \\
> & 4 m^{2}\left[\left(-\left(\lambda O_{1}+O_{3}\right)\left(u^{2}+v^{2}\right)+\left((\gamma+\lambda) O_{1}+2 O_{3}\right) u-\left(\gamma O_{1}+O_{3}\right)\right)^{2}\right. \\
& \left.\left.+(\gamma-\lambda)^{2} O_{1}^{2} v^{2}\right]\right\}, \quad m \geq 0,0<q<1 .
\end{aligned}
$$

We note that

(i) $m-P_{q}(\gamma, \lambda) \subset P(\beta)$, where $\beta$ is given by

$$
\beta=\frac{4 m+(1-\gamma) O_{1}}{4 m+(1-\lambda) O_{1}} \text {. }
$$

(ii) As $q \longrightarrow 1^{-}$, the class $m-P_{q}(\gamma, \lambda)$ becomes the class $m-P(\gamma, \lambda)$ and $\Omega_{q, m}(\gamma, \lambda) \equiv \Omega_{m}(\gamma, \lambda)[22]$.

(iii) When $q \longrightarrow 1^{-}$and $\gamma=1, \lambda=-1$, the class $m-P_{q}(\gamma, \lambda)$ reduces to the class $P\left(p_{m}\right)$ and $\Omega_{q, m}(\gamma, \lambda) \equiv \Omega_{m}[10]$.
Definition 4 (see [20]). Let $g(\varsigma)$ of form (2) be in $\mathscr{A}$ and $\alpha \geq 0, m \in[0, \infty)$. Then, $g \in m-\operatorname{UCV}_{q}(\gamma, \lambda)$ if and only if

$$
\frac{D_{q}\left(\varsigma D_{q} g(\varsigma)\right)}{D_{q} g(\varsigma)}<\frac{\left(O_{1} \gamma+O_{3}\right) p_{m}(\varsigma)-(\gamma-1) O_{1}}{\left(O_{1} \lambda+O_{3}\right) p_{m}(\varsigma)-(\lambda-1) O_{1}}
$$

where $p_{m}(\varsigma)$ is given by (8).

Inspired by the above recent mentioned work, we announce the following novel classes of analytic functions.

Definition 5. Let $f(\varsigma) \in \mathscr{A}, \alpha \geq 0, m \in[0, \infty)$. Then, $f \in m-\mathrm{UM}_{q}(\alpha, \gamma, \lambda)$ if and only if

$$
\operatorname{Re}\left(\frac{(\lambda-1) O_{1} J_{q}(\alpha, f ; \varsigma)-(\gamma-1) O_{1}}{\left(\lambda O_{1}+O_{3}\right) J_{q}(\alpha, f ; \varsigma)-\left(\gamma O_{1}+O_{3}\right)}\right)>m\left|\frac{(\lambda-1) O_{1} J_{q}(\alpha, f ; \varsigma)-(\gamma-1) O_{1}}{\left(\lambda O_{1}+O_{3}\right) J_{q}(\alpha, f ; \varsigma)-\left(\gamma O_{1}+O_{3}\right)}-1\right| .
$$

Equivalently, $f \in m-\mathrm{UM}_{q}(\alpha, \gamma, \lambda)$ if and only if

$$
J_{q}(\alpha, f ; \varsigma)=(1-\alpha) \frac{\varsigma D_{q} f(\varsigma)}{f(\varsigma)}+\alpha \frac{D_{q}\left(\varsigma D_{q} f(\varsigma)\right)}{D_{q} f(\varsigma)} \prec \frac{\left(O_{1} \gamma+O_{3}\right) p_{m}(\varsigma)-(\gamma-1) O_{1}}{\left(O_{1} \lambda+O_{3}\right) p_{m}(\varsigma)-(\lambda-1) O_{1}}
$$


Definition 6. Let $f(\varsigma) \in \mathscr{A}, \alpha \geq 0, m \in[0, \infty)$. Then, $f \in m-\mathrm{UQ}_{q}(\alpha, \gamma, \lambda)$ if and only if there exists an analytic function $g(\varsigma) \in m-\mathrm{UCV}_{q}(\gamma, \lambda)$ such that

$$
\operatorname{Re}\left(\frac{(\lambda-1) O_{1} \mathscr{J}_{q}(\alpha, f ; \varsigma)-(\gamma-1) O_{1}}{\left(\lambda O_{1}+O_{3}\right) \mathscr{J}_{q}(\alpha, f ; \varsigma)-\left(\gamma O_{1}+O_{3}\right)}\right)>m\left|\frac{(\lambda-1) O_{1} \mathscr{J}_{q}(\alpha, f ; \varsigma)-(\gamma-1) O_{1}}{\left(\lambda O_{1}+O_{3}\right) \mathscr{J}_{q}(\alpha, f ; \varsigma)-\left(\gamma O_{1}+O_{3}\right)}-1\right| .
$$

Equivalently, $f \in m-\mathrm{UQ}_{q}(\alpha, \gamma, \lambda)$ if and only if

$$
\mathscr{J}_{q}(\alpha, f ; \varsigma)=(1-\alpha) \frac{D_{q} f(\varsigma)}{D_{q} g(\varsigma)}+\alpha \frac{D_{q}\left(\varsigma D_{q} f(\varsigma)\right)}{D_{q} g(\varsigma)}<\frac{\left(O_{1} \gamma+O_{3}\right) p_{m}(\varsigma)-(\gamma-1) O_{1}}{\left(O_{1} \lambda+O_{3}\right) p_{m}(\varsigma)-(\lambda-1) O_{1}}
$$

We note the following special cases:

(i) When $q \longrightarrow 1^{-}$, the classes $m-\mathrm{UM}_{q}(\alpha, \gamma, \lambda)$ and $m-\mathrm{UQ}_{q}(\alpha, \gamma, \lambda)$ reduce to the classes $m-\operatorname{UM}(\alpha, \gamma, \lambda)$ [21] and $m-\operatorname{UQ}(\alpha, \gamma, \lambda)$ [18], respectively.

(ii) When $q \longrightarrow 1^{-}$and $\alpha=0$, the classes $m-\mathrm{UM}_{q}(\alpha, \gamma, \lambda)$ and $m-\mathrm{UQ}_{q}(\alpha, \gamma, \lambda)$ cut down to the classes $m-\operatorname{UST}(\gamma, \lambda)[22]$ and $m-\operatorname{UKV}(\gamma, \lambda)$ [16].

(iii) When $q \longrightarrow 1^{-}$and $\alpha=1$, the classes $m-\mathrm{UM}_{q}(\alpha, \gamma, \lambda)$ and $m-\mathrm{UQ}_{q}(\alpha, \gamma, \lambda)$ scale down to the classes $m-\operatorname{UCV}(\gamma, \lambda)$ [22] and $m-\mathrm{UC}^{*} V(\gamma, \lambda)[16]$.

(iv) When $\alpha=0$ and $\alpha=1$, the classes $m-\mathrm{UM}_{q}(\alpha, \gamma, \lambda)$ and $m-\mathrm{UQ}_{q}(\alpha, \gamma, \lambda)$ diminish, respectively, to those classes of functions considered in $[17,20]$.

(v) When $\alpha=0=m$ in Definition 2, the class $m-\mathrm{UM}_{q}(\alpha, \gamma, \lambda)$ becomes the class $q$-starlike functions $\operatorname{ST}_{q}(\gamma, \lambda)$ of Janowski type recently explored by Srivastava et al. [28].

To effectively establish our findings, the following set of lemmas is required.

\section{A Set of lemmas}

Lemma 1 (see [4]). Let $m \geq 0$ and $p_{m}(\varsigma)$ given by (8) be of the form $p_{m}(\varsigma)=1+Q_{1} \varsigma+Q_{1} \varsigma^{2}+\cdots$. Then,

$$
Q_{1}= \begin{cases}\frac{8}{\pi^{2}}, \quad m=1, & \\ \frac{2 T^{2}}{1-m^{2}}, \quad 0 \leq m<1, & \\ \frac{\pi^{2}}{4 K^{2}(t)\left(t^{2}-1\right)(1+t) \sqrt{t}}, & m>1,\end{cases}
$$

$$
Q_{2}=\left\{\begin{array}{l}
\frac{2}{3} Q_{1}, \quad m=1, \\
\frac{2+T^{2}}{3} Q_{1}, \quad 0 \leq m<1, \\
\frac{4 K^{2}(t)\left(t^{2}+6 t+1\right)-\pi^{2}}{24 K^{2}(t)(1+t) \sqrt{t}} Q_{1}, \quad m>1,
\end{array}\right.
$$

where $T=\left(2 \cos ^{-1} m\right) / \pi$ and $t \in(0,1)$ is chosen such that $m=\cosh \left(\left(\pi K^{\prime}(t)\right) / K(t)\right)$, where $K(t)$ is Legendre's complete elliptic integral of the first kind.

Lemma 2 (see [17]). If $p(\varsigma)=1+c_{1} \varsigma+c_{2} \varsigma^{2}+\cdots \in P$, then, for any real number $\delta$,

$$
\left|c_{2}-\delta c_{1}^{2}\right| \leq\left\{\begin{array}{l}
-4 \delta+2, \quad \delta \leq 0 \\
2, \quad 0 \leq \delta \leq 1 \\
4 \delta+2, \quad \delta \geq 1
\end{array}\right.
$$


when $\delta<0$ or $\delta>1$, the equality holds if and only if $p(\varsigma)=$ $(1+\varsigma) /(1-\varsigma)$ or one of its rotations. If $0<\delta<1$, then the equality holds if and only if $p(\varsigma)=\left(1+\varsigma^{2}\right) /\left(1-\varsigma^{2}\right)$ or one of its rotations. If $\delta=0$, then the equality holds if and only if

$$
p(\varsigma)=\left(\frac{1+\nu}{2}\right) \frac{1+\varsigma}{1-\varsigma}+\left(\frac{1-\nu}{2}\right) \frac{1-\varsigma}{1+\varsigma}, \quad 0 \leq v \leq 1,
$$

or one of its rotations. If $\delta=1$, then the equality holds if and only if $p(\varsigma)$ is the reciprocal of one of the functions such that equality holds for the case $\delta=0$. Although the above upper bound is sharp, when $0<\delta<1$ it can be improved as follows:

$$
\begin{aligned}
& \left|c_{2}-\delta c_{1}^{2}\right|+\left|c_{1}\right|^{2} \leq 2, \quad\left(0<\delta \leq \frac{1}{2}\right), \\
& \left|c_{2}-\delta c_{1}^{2}\right|+(1-\delta)\left|c_{1}\right|^{2} \leq 2, \quad\left(\frac{1}{2}<\delta \leq 1\right) .
\end{aligned}
$$

Lemma 3 (see [20]). Let $g(\varsigma)$ be of form (2) and $g \in m-U C V_{q}(\gamma, \lambda)$. Then,

$$
\left|b_{n}\right| \leq \frac{1}{[n]_{q}} \prod_{\imath=0}^{n-2}\left(\frac{\left|Q_{1} O_{1}(\gamma-\lambda)-4 q[\iota]_{q}\right|}{4 q[\iota+1]_{q}}\right)
$$

where $Q_{1}$ is defined by (23).

\section{Results and Discussion}

We now turn our attention to the main results of this article.

\subsection{Sufficient Conditions}

Theorem 1. A function $f(\varsigma)$ of form (1) belongs to the class $m-U M_{q}(\alpha, \gamma, \lambda)$ if it satisfies the condition

$$
\phi_{n}(\alpha, m, q, \gamma, \lambda)<|\lambda-\gamma| O_{1},
$$

where

$$
\begin{aligned}
& \phi_{n}(\alpha, m, q, \gamma, \lambda)=\sum_{n=2}^{\infty}\left[4(m+1)\left([n]_{q}-1\right)\left(1+\alpha\left([n]_{q}-1\right)\right)+\mid\left(\lambda O_{1}+O_{3}\right)\left(2[n]_{q}\right.\right. \\
& \left.\left.+\alpha\left([n]_{q}-1\right)^{2}\right)-\left(\gamma O_{1}+O_{3}\right)\left([n]_{q}+1\right) \mid\right]\left|a_{n}\right| \\
& +\sum_{n=2}^{\infty} \sum_{\imath=2}^{n-1}\left[4(m+1)\left|\left([\iota]_{q}-1\right)+\alpha\left([n+1-\iota]_{q}-[\iota]_{q}\right)\right|\right. \\
& +\mid\left(\lambda O_{1}+O_{3}\right)\left([\iota]_{q}+\alpha\left([n+1-\iota]_{q}-[\iota]_{q}\right)\right) \\
& \left.-\left(\gamma O_{1}+O_{3}\right) \mid\right][n+1-\iota]_{q}\left|a_{\iota} a_{n+1-\iota}\right| .
\end{aligned}
$$

Proof. Suppose condition (29) holds. Then, we need to prove

that

$$
m\left|\frac{(\lambda-1) O_{1} J_{q}(\alpha, f ; \varsigma)-(\gamma-1) O_{1}}{\left(\lambda O_{1}+O_{3}\right) J_{q}(\alpha, f ; \varsigma)-\left(\gamma O_{1}+O_{3}\right)}-1\right|-\operatorname{Re}\left(\frac{(\lambda-1) O_{1} J_{q}(\alpha, f ; \varsigma)-(\gamma-1) O_{1}}{\left(\lambda O_{1}+O_{3}\right) J_{q}(\alpha, f ; \varsigma)-\left(\gamma O_{1}+O_{3}\right)}-1\right)
$$

Therefore,

$$
\begin{aligned}
& m\left|\frac{(\lambda-1) O_{1} J_{q}(\alpha, f ; \varsigma)-(\gamma-1) O_{1}}{\left(\lambda O_{1}+O_{3}\right) J_{q}(\alpha, f ; \varsigma)-\left(\gamma O_{1}+O_{3}\right)}-1\right|-\operatorname{Re}\left(\frac{(\lambda-1) O_{1} J_{q}(\alpha, f ; \varsigma)-(\gamma-1) O_{1}}{\left(\lambda O_{1}+O_{3}\right) J_{q}(\alpha, f ; \varsigma)-\left(\gamma O_{1}+O_{3}\right)}-1\right) \\
& \leq(m+1)\left|\frac{(\lambda-1) O_{1} J_{q}(\alpha, f ; \varsigma)-(\gamma-1) O_{1}}{\left(\lambda O_{1}+O_{3}\right) J_{q}(\alpha, f ; \varsigma)-\left(\gamma O_{1}+O_{3}\right)}-1\right| \\
& =4(m+1)\left|\frac{(1-\alpha) \varsigma D_{q} f(\varsigma) D_{q} f(\varsigma)+\alpha f(\varsigma) D_{q}\left(\varsigma D_{q} f(\varsigma)\right)-f(\varsigma) D_{q} f(\varsigma)}{(1-\alpha)\left(\lambda O_{1}+O_{3}\right) \varsigma D_{q} f(\varsigma) D_{q} f(\varsigma)+\alpha\left(\lambda O_{1}+O_{3}\right) f(\varsigma) D_{q}\left(\varsigma D_{q} f(\varsigma)\right)-\left(\gamma O_{1}+O_{3}\right) f(\varsigma) D_{q} f(\varsigma)}\right|
\end{aligned}
$$


We have

$$
\begin{aligned}
\varsigma D_{q} f(\varsigma) D_{q} f(\varsigma) & =\left(\sum_{n=0}^{\infty}[n]_{q} a_{n} \varsigma^{n}\right)\left(\sum_{n=0}^{\infty}[n]_{q} a_{n} \varsigma^{n-1}\right), \quad a_{0}=0, a_{1}=[0]_{q}=[1]_{q}=1 \\
& =\sum_{n=0}^{\infty}\left(\sum_{\iota=0}^{n}[\iota]_{q}[n-\iota]_{q} a_{\iota} a_{n-\iota}\right) \varsigma^{n-1} \\
& =\varsigma+\sum_{n=2}^{\infty}\left(\sum_{\imath=1}^{n+1}[\iota]_{q}[n+1-\iota]_{q} a_{\iota} a_{n+1-\iota}\right) \varsigma^{n} \\
& =\varsigma+\sum_{n=2}^{\infty}\left(2[n]_{q} a_{n}+\sum_{\iota=2}^{n-1}[\iota]_{q}[n+1-\iota]_{q} a_{\iota} a_{n+1-\iota}\right) \varsigma^{n} .
\end{aligned}
$$

Similarly,

$$
\begin{aligned}
& D_{q}\left(\varsigma D_{q} f(\varsigma)\right)=\varsigma+\sum_{n=2}^{\infty}\left(\left([n]_{q}^{2}+1\right) a_{n}+\sum_{\imath=2}^{n-1}[n+1-\iota]_{q}^{2} a_{\iota} a_{n+1-\iota}\right) \varsigma^{n}, \\
& f(\varsigma) D_{q} f(\varsigma)=\varsigma+\sum_{n=2}^{\infty}\left(\left([n]_{q}+1\right) a_{n}+\sum_{\imath=2}^{n-1}[n+1-\iota]_{q} a_{\iota} a_{n+1-\iota}\right) \varsigma^{n} .
\end{aligned}
$$

From inequality (32) and equations (33) and (34), we arrive at

$$
\begin{aligned}
& m\left|\frac{(\lambda-1) O_{1} J_{q}(\alpha, f ; \varsigma)-(\gamma-1) O_{1}}{\left(\lambda O_{1}+O_{3}\right) J_{q}(\alpha, f ; \varsigma)-\left(\gamma O_{1}+O_{3}\right)}-1\right|-\operatorname{Re}\left(\frac{(\lambda-1) O_{1} J_{q}(\alpha, f ; \varsigma)-(\gamma-1) O_{1}}{\left(\lambda O_{1}+O_{3}\right) J_{q}(\alpha, f ; \varsigma)-\left(\gamma O_{1}+O_{3}\right)}-1\right) \\
& \leq 4(m+1) \mid\left\{\sum _ { n = 2 } ^ { \infty } \left[\left([n]_{q}-1\right)\left(1+\alpha\left([n]_{q}-1\right)\right) a_{n}+\sum_{\imath=2}^{n-1}\left(\left([\iota]_{q}-1\right)+\alpha\left([n+1-\iota]_{q}-[\iota]_{q}\right)\right)\right.\right. \\
& \left.\left.\cdot[n+1-\iota]_{q} a_{\iota} a_{n+1-\iota}\right] \varsigma^{n}\right\} \times\left\{(\lambda-\gamma) O_{1} \varsigma+\sum_{n=2}^{\infty}\left[\left(\left(\lambda O_{1}+O_{3}\right)\left(2[n]_{q}+\alpha\left([n]_{q}-1\right)^{2}\right)\right)\right.\right. \\
& \left.-\left(\gamma O_{1}+O_{3}\right)\left([n]_{q}+1\right)\right) a_{n}+\sum_{\imath=2}^{n-1}\left(\left(\lambda O_{1}+O_{3}\right)\left([\iota]_{q}+\alpha\left([n+1-\iota]_{q}-[\iota]_{q}\right)\right)\right. \\
& \left.\left.\left.-\left(\gamma O_{1}+O_{3}\right)\right)[n+1-\iota]_{q} a_{\iota} a_{n+1-\iota}\right] \varsigma^{n}\right\}^{-1} \mid \\
& \leq 4(m+1)\left[\sum_{n=2}^{\infty}\left([n]_{q}-1\right)\left(1+\alpha\left([n]_{q}-1\right)\right)\left|a_{n}\right|+\sum_{\imath=2}^{n-1}\left|\left([\iota]_{q}-1\right)+\alpha\left([n+1-\iota]_{q}-[\iota]_{q}\right)\right|\right. \\
& \left.\cdot[n+1-\iota]_{q}\left|a_{\iota} a_{n+1-\iota}\right|\right] \times\left[|(\lambda-\gamma)| O_{1}-\sum_{n=2}^{\infty} \mid\left(\lambda O_{1}+O_{3}\right)\left(2[n]_{q}+\alpha\left([n]_{q}-1\right)^{2}\right)\right. \\
& \left.-\left(\gamma O_{1}+O_{3}\right)\left([n]_{q}+1\right)|| a_{n}\left|-\sum_{\imath=2}^{n-1}\right|\left(\lambda O_{1}+O_{3}\right)\left([\iota]_{q}+\alpha\left([n+1-\iota]_{q}-[\iota]_{q}\right)\right)-\left(\gamma O_{1}+O_{3}\right)\left|[n+1-\iota]_{q}\right| a_{\iota} a_{n+1-\iota} \mid\right]^{-1} .
\end{aligned}
$$


The last inequality is bounded by 1 if (29) is satisfied. This completes the proof. [21].

As $q \longrightarrow 1^{-}$in Theorem 1 , we are led to Theorem 1 in
Corollary 1. A function $f(\varsigma)$ of type (1) is in $m-U M(\alpha, \gamma, \lambda)$ if it satisfies the condition

$$
\phi_{n}(\alpha, m, \gamma, \lambda)<|\lambda-\gamma|,
$$

where

$$
\begin{aligned}
\phi_{n}(\alpha, m, \gamma, \lambda)= & \sum_{n=2}^{\infty}\left[2(m+1)(n-1)(1+\alpha(n-1))+\mid(\lambda+1)\left(2 n+\alpha(n-1)^{2}\right)\right. \\
& -(\gamma+1)(n+1) \mid]\left|a_{n}\right|+\sum_{n=2}^{\infty} \sum_{\iota=2}^{n-1}[2(m+1)|(\iota-1)+\alpha(n+1-2 \iota)| \\
& +|(\lambda+1)(\iota+\alpha(n+1-2 \iota))-(\gamma+1)|](n+1-\iota)\left|a_{\iota} a_{n+1-\iota}\right| .
\end{aligned}
$$

Setting $\alpha=0$ in Theorem 1, we obtain a variance version of the result presented in [17].
Corollary 2. If $f(\varsigma)$ having representation (1) satisfies the condition

$$
\sum_{n=2}^{\infty}\left[4 q(m+1)[n-1]_{q}+\left|\left(\lambda O_{1}+O_{3}\right)[n]_{q}-\left(\gamma O_{1}+O_{3}\right)\right|\right]\left|a_{n}\right|<|\lambda-\gamma| O_{1}
$$

then $f \in m-U S T_{q}(\gamma, \lambda)$.

If we choose $\alpha=0$ and allow $q \longrightarrow 1^{-}$in the above theorem, our investigation comes down to Theorem 1 in [22].

Corollary 3. A function $f(\varsigma)$ demonstrated by (1) is in the class $m-\operatorname{UST}(\gamma, \lambda)$ if it satisfies the condition

$$
\sum_{n=2}^{\infty}[2(m+1)(n-1)+|(\lambda+1) n-(\gamma+1)|]\left|a_{n}\right|<|\lambda-\gamma| \text {. }
$$

If we choose $\alpha=0, \lambda=-1$, and $\gamma=1$ and allow $q \longrightarrow 1^{-}$ in the above theorem, our investigation comes down to Theorem 1 in [22].

Corollary 4 (see [11]). If $f(\varsigma) \in \mathscr{A}$ satisfies the condition

$$
\sum_{n=2}^{\infty}(m(n-1)+n)\left|a_{n}\right|<1
$$

then $f \in m-U S T_{q \longrightarrow 1^{-}}(0,1,-1)=m-U S T$.

Theorem 2. Let $f \in \mathscr{A}$. Then, $f \in m-U Q_{q}(\alpha, \gamma, \lambda)$ if inequality (42) is satisfied,

$$
\begin{aligned}
& \sum_{n=2}^{\infty} \prod_{\imath=0}^{n-2} \frac{\left(4(m+1)+\left(\gamma O_{1}+O_{3}\right)\right)\left|Q_{1} O_{1}(\gamma-\lambda)-4 q[\iota]_{q} \lambda\right|}{4 q[\iota+1]_{q}} \\
& \quad+\sum_{n=2}^{\infty}\left(4(m+1)+\left(\lambda O_{1}+O_{3}\right)\right)\left(1+\alpha q[n-1]_{q}\right)[n]_{q}\left|a_{n}\right|<|\lambda-\gamma| O_{1}
\end{aligned}
$$

where $Q_{1}$ is given by (23).

Proof. Assuming (42) holds, then it suffices to establish that

$m\left|\frac{(\lambda-1) O_{1} \mathscr{J}_{q}(\alpha, f ; \varsigma)-(\gamma-1) O_{1}}{\left(\lambda O_{1}+O_{3}\right) \mathscr{J}_{q}(\alpha, f ; \varsigma)-\left(\gamma O_{1}+O_{3}\right)}-1\right|-\operatorname{Re}\left(\frac{(\lambda-1) O_{1} \mathscr{J}_{q}(\alpha, f ; \varsigma)-(\gamma-1) O_{1}}{\left(\lambda O_{1}+O_{3}\right) \mathscr{J}_{q}(\alpha, f ; \varsigma)-\left(\gamma O_{1}+O_{3}\right)}-1\right)$ 
Following the same process in the proof of Theorem 1, we have

$$
\begin{aligned}
& m\left|\frac{(\lambda-1) O_{1} \mathscr{J}_{q}(\alpha, f ; \varsigma)-(\gamma-1) O_{1}}{\left(\lambda O_{1}+O_{3}\right) \mathscr{J}_{q}(\alpha, f ; \varsigma)-\left(\gamma O_{1}+O_{3}\right)}-1\right|-\operatorname{Re}\left(\frac{(\lambda-1) O_{1} \mathscr{J}_{q}(\alpha, f ; \varsigma)-(\gamma-1) O_{1}}{\left(\lambda O_{1}+O_{3}\right) \mathscr{J}_{q}(\alpha, f ; \varsigma)-\left(\gamma O_{1}+O_{3}\right)}-1\right) \\
& \leq 4(m+1)\left|\frac{(1-\alpha) D_{q} f(\varsigma)+\alpha D_{q}\left(\varsigma D_{q} f(\varsigma)\right)-D_{q} g(\varsigma)}{\left(\lambda O_{1}+O_{3}\right)\left((1-\alpha) D_{q} f(\varsigma)+\alpha D_{q}\left(\varsigma D_{q} f(\varsigma)\right)\right)-\left(\gamma O_{1}+O_{3}\right) D_{q} g(\varsigma)}\right| \\
& \left(\text { where } g \in m-\operatorname{UCV}_{q}(\gamma, \lambda)\right) \\
& \leq \frac{4(m+1)\left(\sum_{n=2}^{\infty}\left(1+\alpha\left([n]_{q}-1\right)\right)[n]_{q}\left|a_{n}\right|+[n]_{q}\left|b_{n}\right|\right)}{|\lambda-\gamma| O_{1}-\sum_{n=2}^{\infty}\left|\left(\lambda O_{1}+O_{3}\right)\left(1+\alpha\left([n]_{q}-1\right)\right)\right|[n]_{q}-\left(\gamma O_{1}+O_{3}\right) \sum_{n=2}^{\infty}[n]_{q}\left|b_{n}\right|} \\
& \leq \frac{4(m+1)\left[\sum_{n=2}^{\infty}\left(1+\alpha\left([n]_{q}-1\right)\right)[n]_{q}\left|a_{n}\right|+\sum_{n=2}^{\infty} \prod_{l=0}^{n-2}\left(\left|Q_{1} O_{1}(\gamma-\lambda)-4 q[\iota]_{q}\right| / 4 q[\iota+1]_{q}\right)\right]}{|\lambda-\gamma| O_{1}-\sum_{n=2}^{\infty}\left(\lambda O_{1}+O_{3}\right)\left(1+\alpha\left([n]_{q}-1\right)\right)[n]_{q}\left|a_{n}\right|-\left(\gamma O_{1}+O_{3}\right) \sum_{n=2}^{\infty} \prod_{l=0}^{n-2}\left(\left|Q_{1} O_{1}(\gamma-\lambda)-4 q[\iota]_{q}\right| / 4 q[\iota+1]_{q}\right)}
\end{aligned}
$$

where we have used Lemma 3. Thus, the last inequality is bounded by 1 if (42) is satisfied. Hence, we complete the proof.

As $q \longrightarrow 1^{-}$in Theorem 2 , we obtain the similar result proved in [18].

Corollary 5. Let $f \in \mathscr{A}$. Then, $f \in m-U Q(\alpha, \gamma, \lambda)$ if

$$
\begin{aligned}
& \sum_{n=2}^{\infty} \prod_{\iota=0}^{n-2} \frac{(2(m+1)+(\gamma+1))\left|\mathcal{Q}_{1} O_{1}(\gamma-\lambda)-2 \iota \lambda\right|}{2(\iota+1)} \\
& \quad+\sum_{n=2}^{\infty}(2(m+1)+(\lambda+1))(1+\alpha(n-1)) n\left|a_{n}\right|<|\lambda-\gamma|,
\end{aligned}
$$

holds.

For $\alpha=0$ in Theorem 2, we get the result established by Naeem et al. [20].

Corollary 6. Let $f \in \mathscr{A}$. Then, $f \in m-U K V_{q}(\gamma, \lambda)$ if inequality (46) is satisfied,

$$
\begin{aligned}
& \sum_{n=2}^{\infty} \prod_{\imath=0}^{n-2} \frac{\left(4(m+1)+\left(\gamma O_{1}+O_{3}\right)\right)\left|Q_{1} O_{1}(\gamma-\lambda)-4 q[\iota]_{q} \lambda\right|}{4 q[\iota+1]_{q}} \\
& \quad+\sum_{n=2}^{\infty}\left(4(m+1)+\left(\lambda O_{1}+O_{3}\right)\right)[n]_{q}\left|a_{n}\right|<|\lambda-\gamma| O_{1} .
\end{aligned}
$$

For $\alpha=0$ and $\alpha=1$ as $q \longrightarrow 1^{-}$in Theorem 2, we obtain the following results established in [16].

Corollary 7. Let $f \in \mathscr{A}$. Then, $f \in m-U K V(\gamma, \lambda)$ if

$$
\sum_{n=2}^{\infty} \prod_{l=0}^{n-2} \frac{(2(m+1)+(\gamma+1))\left|Q_{1} O_{1}(\gamma-\lambda)-2 \iota \lambda\right|}{2(\iota+1)}
$$

$$
+\sum_{n=2}^{\infty}(2(m+1)+(\lambda+1)) n\left|a_{n}\right|<|\lambda-\gamma|
$$

is satisfied.

Corollary 8. Let $f \in \mathscr{A}$. Then, $f \in m-U K V(\gamma, \lambda)$ if 


$$
\begin{gathered}
\sum_{n=2}^{\infty} \prod_{\iota=0}^{n-2} \frac{(2(m+1)+(\gamma+1))\left|Q_{1} O_{1}(\gamma-\lambda)-2 \iota \lambda\right|}{2(\iota+1)} \\
+\sum_{n=2}^{\infty}(2(m+1)+(\lambda+1)) n^{2}\left|a_{n}\right|<|\lambda-\gamma|,
\end{gathered}
$$

is satisfied.

\subsection{Fekete Szegö Inequality}

Theorem 3. Let $f \in m-U M_{q}(\alpha, \gamma, \lambda)$. Then, for any real number $\delta$, we have

$$
\left|a_{3}-\delta a_{2}^{2}\right| \leq \frac{(\gamma-\lambda) \mathscr{Q}_{1}}{8 q\left(1+\alpha q[2]_{q}\right)} \times \begin{cases}\Lambda_{1}(\alpha, m, q, \gamma, \lambda), & \delta<\rho_{1}, \\ 2, \quad \rho_{1} \leq \delta \leq \rho_{2}, & \\ \Lambda_{2}(\alpha, m, q, \gamma, \lambda), \quad \delta>\rho_{2},\end{cases}
$$

where

$$
\begin{aligned}
& \rho_{1}=\frac{1}{[2]_{q}\left(1+\alpha q[2]_{q}\right)}\left[\frac{8 q(1+\alpha q)^{2}}{(\gamma-\lambda) O_{1} Q_{1}}\left(\frac{Q_{2}-Q_{1}}{2 Q_{1}}-\frac{\left(\lambda O_{1}+O_{3}\right) Q_{1}}{8}\right)+\left(1+\alpha q\left([2]_{q}+1\right)\right)\right] \\
& \rho_{2}=\frac{1}{[2]_{q}\left(1+\alpha q[2]_{q}\right)}\left[\frac{8 q(1+\alpha q)^{2}}{(\gamma-\lambda) O_{1} Q_{1}}\left(\frac{Q_{2}+Q_{1}}{2 Q_{1}}-\frac{\left(\lambda O_{1}+O_{3}\right) Q_{1}}{8}\right)+\left(1+\alpha q\left([2]_{q}+1\right)\right)\right], \\
& \Lambda_{1}(\alpha, m, q, \gamma, \lambda)=\frac{4 Q_{2}-\left(\lambda O_{1}+O_{3}\right) Q_{1}^{2}}{2 Q_{1}}+\frac{\left(\left(1+\alpha q\left([2]_{q}+1\right)\right)-\delta[2]_{q}\left(1+\alpha q[2]_{q}\right)\right)(\gamma-\lambda) O_{1} Q_{1}}{2 q(1+\alpha q)^{2}} \\
& \Lambda_{2}(\alpha, m, q, \gamma, \lambda)=\frac{\left(\lambda O_{1}+O_{3}\right) Q_{1}^{2}-4 Q_{2}}{2 Q_{1}}+\frac{\left(\delta[2]_{q}\left(1+\alpha q[2]_{q}\right)-\left(1+\alpha q\left([2]_{q}+1\right)\right)\right)(\gamma-\lambda) O_{1} Q_{1}}{2 q(1+\alpha q)^{2}} \\
& J_{q}(\alpha, f ; \varsigma)=\frac{\left(O_{1} \gamma+O_{3}\right) p_{m}(w(\varsigma))-(\gamma-1) O_{1}}{\left(O_{1} \lambda+O_{3}\right) p_{m}(w(\varsigma))-(\lambda-1) O_{1}} . \\
& w(\varsigma)=\frac{p(\varsigma)-1}{p(\varsigma)+1}=\frac{c_{1}}{2} \varsigma+\frac{c_{2}-c_{1}^{2}}{2} \varsigma^{2}+\cdots, \\
& p_{m}(\varsigma)=1+\frac{c_{1} Q_{1}}{2} \varsigma+\left(\frac{\left(2 c_{2}-c_{1}^{2}\right) Q_{1}+c_{1}^{2} Q_{2}}{4}\right) \varsigma^{2}+\cdots
\end{aligned}
$$

Thus,

Using the representation for $p_{m}(\varsigma)$ in Lemma 3 and the relationship between $w(\varsigma)$ and $p(\varsigma)=1+c_{1} \varsigma+c_{2} \varsigma^{2}+\cdots \in P$, we can write

$$
\begin{aligned}
J_{q}(\alpha, f ; \varsigma) & =\frac{4+\left(\left(\left(\gamma O_{1}+O_{3}\right) c_{1} Q_{1}\right) / 2\right) \varsigma+\left(\left(\gamma O_{1}+O_{3}\right) / 4\right)\left[\left(2 c_{2}-c_{1}^{2}\right) Q_{1}+c_{2}^{1} Q_{2}\right] \varsigma^{2}+\cdots}{4+\left(\left(\left(\lambda O_{1}+O_{3}\right) c_{1} Q_{1}\right) / 2\right) \varsigma+\left(\left(\lambda O_{1}+O_{3}\right) / 4\right)\left[\left(2 c_{2}-c_{1}^{2}\right) Q_{1}+c_{2}^{1} Q_{2}\right] \varsigma^{2}+\cdots} \\
& =1+\frac{(\gamma-\lambda) c_{1} O_{1} Q_{1}}{8} \varsigma+\left[\left(\frac{\gamma-\lambda}{16}\right) O_{1}\left(\left(2 c_{2}-c_{1}^{2}\right) Q_{1}+c_{1}^{2} Q_{2}\right)\right. \\
& \left.-\frac{(\gamma-\lambda)\left(\lambda O_{1}+O_{3}\right) c_{1}^{2} O_{1} Q_{1}^{2}}{64}\right] \varsigma^{2}+\cdots .
\end{aligned}
$$


However,

$$
\begin{aligned}
J_{q}(\alpha, f ; \varsigma) & =(1-\alpha) \frac{\varsigma D_{q} f(\varsigma)}{f(\varsigma)}+\alpha \frac{D_{q}\left(\varsigma D_{q} f(\varsigma)\right)}{D_{q} f(\varsigma)} \\
& =1+q(1+\alpha q) a_{2} \varsigma+q\left[[2]_{q}\left(1+\alpha q[2]_{q}\right) a_{3}-\left(1+\alpha q\left([2]_{q}+1\right)\right) a_{2}^{2}\right] \varsigma^{2}+\cdots
\end{aligned}
$$

On comparing the coefficients of $\varsigma$ and $\varsigma^{2}$ of (53) and (54), we obtain

$$
\begin{aligned}
a_{2} & =\frac{(\gamma-\lambda) c_{1} O_{1} Q_{1}}{8 q(1+\alpha q)}, \\
a_{3} & =\frac{1}{q[2]_{q}\left(1+\alpha q[2]_{q}\right)}\left[\left(\frac{\gamma-\lambda}{16}\right) O_{1}\left(\left(2 c_{2}-c_{1}^{2}\right) Q_{1}+c_{1}^{2} Q_{2}\right)-\frac{\left.(\gamma-\lambda)\left(\lambda O_{1}+O_{3}\right) c_{1}^{2} O_{1} Q_{1}^{2}\right]}{64}\right] \\
& +\frac{\left(1+\alpha q\left([2]_{q}+1\right)\right) a_{2}^{2}}{q[2]_{q}\left(1+\alpha q[2]_{q}\right)} .
\end{aligned}
$$

Now, for a real number $\delta$, we have

$$
\begin{aligned}
\left|a_{3}-\delta a_{2}^{2}\right| & =\mid \frac{1}{q[2]_{q}\left(1+\alpha q[2]_{q}\right)}\left(\frac{\gamma-\lambda}{8}\right) O_{1}\left[Q_{1} c_{2}+\left(\frac{Q_{2}-Q_{1}}{2}-\frac{\left(\lambda O_{1}+O_{3}\right) Q_{1}^{2}}{8}\right) c_{1}^{2}\right] \\
& +(\gamma-\lambda) O_{1} Q_{1}^{2} c_{1}^{2}\left(\frac{\gamma-B}{8}\right) O_{1} \frac{\left[\left(1+\alpha q\left([2]_{q}+1\right)\right)-\delta[2]_{q}\left(1+\alpha q[2]_{q}\right)\right]}{8 q^{2}(1+\alpha q)^{2}[2]_{q}\left(1+\alpha q[2]_{q}\right)} \mid \\
& =\frac{(\gamma-\lambda) O_{1} Q_{1}}{8 q[2]_{q}\left(1+\alpha q[2]_{q}\right)} \mid c_{2}-\left[\frac{Q_{1}-Q_{2}}{2 Q_{1}}+\frac{\left(\lambda O_{1}+O_{3}\right) Q_{1}}{8}\right. \\
& \left.-\frac{\left.\left(\left(1+\alpha q\left([2]_{q}+1\right)\right)-\delta[2]_{q}\left(1+\alpha q[2]_{q}\right)\right)(\gamma-\lambda) O_{1} Q_{1}\right]}{8 q(1+\alpha q)^{2}}\right] c_{1}^{2} \mid \\
& =\frac{(\gamma-\lambda) O_{1} Q_{1}}{8 q[2]_{q}\left(1+\alpha q[2]_{q}\right)}\left|c_{2}-\beta c_{1}^{2}\right|,
\end{aligned}
$$

where

$$
\beta=\frac{\mathscr{Q}_{1}-\mathscr{Q}_{2}}{2 Q_{1}}+\frac{\left(\lambda O_{1}+O_{3}\right) Q_{1}}{8}-\frac{\left[\left(1+\alpha q\left([2]_{q}+1\right)\right)-\delta[2]_{q}\left(1+\alpha q[2]_{q}\right)\right](\gamma-\lambda) O_{1} Q_{1}}{8 q(1+\alpha q)^{2}}
$$

Hence, the result follows from Lemma 2.

If $\alpha=0$ and $0<m<1$, then Theorem 3 reduces to Theorem 10 in [17].
Corollary 9. Let $0<m<1$ and $f \in m-U S T_{q}(\gamma, \lambda)$ be of form (1). Then, for any real number $\delta$, we have 


$$
\left|a_{3}-\delta a_{2}^{2}\right| \leq \frac{(\gamma-\lambda) T^{2}}{4 q\left(1-m^{2}\right)} \times\left\{\begin{array}{l}
\Lambda_{1}(m, q, \gamma, \lambda), \quad \delta<\lambda_{1}, \quad \text { where } \\
2, \quad \lambda_{1} \leq \delta \leq \lambda_{2}, \\
\Lambda_{2}(m, q, \gamma, \lambda), \quad \delta>\lambda_{2},
\end{array}\right.
$$

$$
\lambda_{1}=\frac{4 q\left(1-m^{2}\right)}{(\gamma-\lambda)(1+q)^{2} T^{2}}\left(\frac{T^{2}-1}{6}-\frac{T^{2}(\lambda(1+q)+3-q)}{4\left(1-m^{2}\right)}\right)+\frac{1}{1+q},
$$

where

$$
\begin{gathered}
\lambda_{2}=\frac{4 q\left(1-m^{2}\right)}{(\gamma-\lambda)(1+q)^{2} T^{2}}\left(\frac{T^{2}+5}{6}-\frac{T^{2}(\lambda(1+q)+3-q)}{4\left(1-m^{2}\right)}\right)+\frac{1}{1+q}, \\
\Lambda_{1}(m, q, \gamma, \lambda)=\frac{2\left(2+T^{2}\right)}{3}-\frac{T^{2}(\lambda(1+q)+3-q)}{\left(1-m^{2}\right)}+\frac{T^{2}(1-\delta(1+q))(1+q)(\gamma-\lambda)}{q\left(1-m^{2}\right)}, \\
\Lambda_{2}(m, q, \gamma, \lambda)=\frac{T^{2}(\lambda(1+q)+3-q)}{\left(1-m^{2}\right)}-\frac{2\left(2+T^{2}\right)}{3}+\frac{T^{2}(\delta(1+q)-1)(1+q)(\gamma-\lambda)}{q\left(1-m^{2}\right)} .
\end{gathered}
$$

The result is sharp.

Proof. The proof is straightforward from Theorem 3 and Lemma 1.

If $\alpha=0$ in Theorem 3, the case $m=1$ is contained in the following corollary.

Corollary 10. Let $m=1$, and $f(\varsigma)$ of form (1) belongs to $m-U S T_{q}(\gamma, \lambda)$. Then, for a real number $\delta$, we have the following sharp inequality:

$$
\left|a_{3}-\delta a_{2}^{2}\right| \leq \frac{(\gamma-\lambda)}{q \pi^{2}} \times\left\{\begin{array}{l}
\Lambda_{1}(q, \gamma, \lambda), \quad \delta<\nu_{1} \\
2, \quad v_{1} \leq \delta \leq \nu_{2} \\
\Lambda_{2}(q, \gamma, \lambda), \quad \delta>v_{2}
\end{array}\right.
$$

where

$$
v_{1}=\frac{1}{1+q}-\frac{q \pi^{2}}{(\gamma-\lambda)(1+q)^{2}}\left(\frac{1}{6}+\frac{(\lambda(1+q)+3-q)}{\pi^{2}}\right),
$$

where

$$
\begin{gathered}
\nu_{2}=\frac{1}{1+q}-\frac{q \pi^{2}}{(\gamma-\lambda)(1+q)^{2}}\left(\frac{5}{6}-\frac{(\lambda(1+q)+3-q)}{\pi^{2}}\right), \\
\Lambda_{1}(q, \gamma, \lambda)=\frac{4}{3}-\frac{4(\lambda(1+q)+3-q)}{\pi^{2}}+\frac{(1-\delta(1+q))(1+q)(\gamma-\lambda)}{q \pi^{2}}, \\
\Lambda_{2}(m, q, \gamma, \lambda)=\frac{4(\lambda(1+q)+3-q)}{\pi^{2}}-\frac{4}{3}+\frac{(\delta(1+q)-1)(1+q)(\gamma-\lambda)}{q \pi^{2}} .
\end{gathered}
$$


The result is sharp.

Theorem 3 becomes Theorem 1 in [28] when $\alpha=m=0$.
Corollary 11. Let $f(\varsigma)$ of the series representation (1) be in $S T_{q}(\gamma, \lambda)$. Then, we have the sharp inequality:

$$
\left|a_{3}-\delta a_{2}^{2}\right| \leq\left(\frac{\gamma-\lambda}{4 q^{2}}\right) \times \begin{cases}(\gamma-\lambda)+(\gamma-2 \lambda-1) q+(1-\lambda) q^{2}-\delta(\gamma-\lambda)(1+q)^{2}, & \delta<\sigma_{1}, \\ 2 q, & \sigma_{1} \leq \delta \leq \sigma_{2}, \\ (\lambda-\gamma)+(2 \lambda-\gamma+1) q+(\lambda-1) q^{2}+\delta(\gamma-\lambda)(1+q)^{2}, & \delta>\sigma_{2}\end{cases}
$$

where

$$
\begin{aligned}
& \sigma_{1}=\frac{1}{1+q}\left(1-\frac{q(\lambda(1+q)+3-q)}{(\gamma-\lambda)(1+q)}\right), \\
& \sigma_{2}=\sigma_{1}+\frac{4 q}{(\gamma-\lambda)(1+q)^{2}} .
\end{aligned}
$$

Thus, we have the result.

\subsection{Covering Theorems}

Theorem 4. The range of every univalent functions $f \in m-$ $U_{q}(\alpha, \gamma, \lambda)$ contains the disc:

$$
|\varsigma|<\frac{4 q(1+\alpha q)}{8 q(1+\alpha q)+(\gamma-\lambda) O_{1}\left|Q_{1}\right|} .
$$

Proof. From the proof of Theorem 3, we can see that

$$
\left|a_{2}\right| \leq \frac{(\gamma-\lambda) O_{1}\left|Q_{1}\right|}{4 q(1+\alpha q)} .
$$

Since the Koebe one-quater theorem asserted that each omitted value $w$ of the univalent function $f(\varsigma)$ of form (1) satisfies

$$
\begin{aligned}
|w| & >\frac{1}{2+\left|a_{2}\right|} \\
& \geq \frac{4 q(1+\alpha q)}{8 q(1+\alpha q)+(\gamma-\lambda) O_{1}\left|Q_{1}\right|} .
\end{aligned}
$$

Corollary 12. The range of every univalent function $f \in S T_{q}(\gamma, \lambda)$ contains the disc:

$$
|\varsigma|<\frac{2 q}{4 q+(\gamma-\lambda) O_{1}} .
$$

Theorem 5. The range of every univalent function $f \in m-$ $U Q_{q}(\alpha, \gamma, \lambda)$ contains the same disc given by (66).

Proof. Let $w(\varsigma)$ be a Schwarz function. We note first in Theorem 3 that

$$
\frac{\left(O_{1} \gamma+O_{3}\right) p_{m}(w(\varsigma))-(\gamma-1) O_{1}}{\left(O_{1} \lambda+O_{3}\right) p_{m}(w(\varsigma))-(\lambda-1) O_{1}}=1+\gamma_{1} \varsigma+\gamma_{2} \varsigma^{2}+\cdots,
$$

where

$$
\begin{aligned}
& \gamma_{1}=\frac{(\gamma-\lambda) c_{1} O_{1} Q_{1}}{8}, \\
& \gamma_{2}=\left(\frac{\gamma-\lambda}{16}\right) O_{1}\left(\left(2 c_{2}-c_{1}^{2}\right) Q_{1}+c_{1}^{2} Q_{2}\right)-\frac{(\gamma-\lambda)\left(\lambda O_{1}+O_{3}\right) c_{1}^{2} O_{1} Q_{1}^{2}}{64} .
\end{aligned}
$$

Since $f \in m-\mathrm{UQ}_{q}(\alpha, \gamma, \lambda)$, then for some $g(\varsigma)=\varsigma+b_{2} \varsigma^{2}+b_{3} \varsigma^{3}+\cdots \in m-\mathrm{UCV}_{q}(\gamma, \lambda)$, we have

$$
(1-\alpha) D_{q} f(\varsigma)+\alpha D_{q}\left(\varsigma D_{q} f(\varsigma)\right)=\left(\frac{\left(O_{1} \gamma+O_{3}\right) p_{m}(w(\varsigma))-(\gamma-1) O_{1}}{\left(O_{1} \lambda+O_{3}\right) p_{m}(w(\varsigma))-(\lambda-1) O_{1}}\right) D_{q} g(\varsigma)
$$

which in turn implies

$$
1+[2]_{q}(1+\alpha q) a_{2} \varsigma+\cdots=1+\left(\gamma_{1}+[2]_{q} b_{2}\right) \varsigma \cdots .
$$

It is easy to see that

$$
b_{2}=\frac{\gamma_{1}}{q[2]_{q}} .
$$

Therefore, comparing the coefficients of $\varsigma$ of (73) and applying (74), we obtain

$$
a_{2}=\frac{\gamma_{1}}{q(1+\alpha q)}
$$

such that 


$$
\left|a_{2}\right| \leq \frac{(\gamma-\lambda) O_{1}\left|Q_{1}\right|}{4 q(1+\alpha q)} .
$$

Now, proceeding the same way as in the proof of Theorem 4, we have the required result.

\section{Conclusion}

Using the concept of $q$-calculus, we have introduced some new subclasses of analytic functions in the unit disc related to Janowski class of functions. In addition, sufficient conditions, Fekete-Szegö inequality as well as covering results for functions belonging to these new classes were established. Consequently, many remarkable special cases of our findings which were studied in the previous work were obtained $[19,26]$.

\section{Data Availability}

No data were used to support the findings of the study.

\section{Conflicts of Interest}

The authors declare that they have no conflicts of interest.

\section{Acknowledgments}

The authors would like to thank the Rector of COMSATS University Islamabad, Islamabad, Pakistan, for providing excellent research and academic environment. Maslina Darus was supported by the Grant GUP-2019-032.

\section{References}

[1] S. Agrawal and S. K. Sahoo, "A generalization of starlike functions of order alpha," Hokkaido Mathematical Journal, vol. 46, no. 1, pp. 15-27, 2017.

[2] A. Aral, V. Gupta, and R. P. Agarwal, Applications of q-calculus in Operator Theory, Springer, New York, NY, USA, 2013.

[3] A. W. Goodman, "On uniformly convex functions," Annales Polonici Mathematici, vol. 56, no. 1, pp. 87-92, 1991.

[4] S. Hussain, S. Khan, M. A. Zaighum, and M. Darus, "Applications of a $q$-Salagean type operator on multivalent functions," Journal of Inequalities and Applications, vol. 2018, no. 1, 12 pages, 2008.

[5] M. E. H. Ismail, E. Merkes, and D. Styer, "A generalization of starlike functions, Complex Var," Complex Variables, Theory and Application, vol. 14, no. 1-4, pp. 77-84, 1990.

[6] D. O. Jackson, T. Fukuda, O. Dunn, and E. Majors, "On q-definite integrals," The Quarterly Journal of Pure and Applied Mathematics, vol. 41, pp. 193-203, 1910.

[7] W. Janowski, "Some extremal problems for certain families of analytic functions I," Annales Polonici Mathematici, vol. 28, pp. 297-326.

[8] S. Kanas and A. Wisniowska, "Conic regions and $k$-uniform convexity, II," Zeszyty Naukowe Politechniki Rzeszowskiej. Matematyka, vol. 170, pp. 65-78, 1998.

[9] S. Kanas and A. Wisniowska, "Uniformly starlike and convex functions and other related classes of univalent functions," Annales Universitatis Mariae Curie-Sklodowska Section A, vol. 53, pp. 95-105, 1999.
[10] S. Kanas and A. Wisniowska, "Conic regions and k-uniform convexity," Journal of Computational and Applied Mathematics, vol. 105, no. 1-2, pp. 327-336, 1999.

[11] S. Kanas and A. Wisniowska, "Conic domains and starlike functions," Revue Roumaine des Mathematiques Pures et Appliquees, vol. 45, no. 4, pp. 647-658, 2000.

[12] S. Kanas and H. M. Srivastava, "Linear operators associated with k-uniformly convex functions," Integral Transforms and Special Functions, vol. 9, no. 2, pp. 121-132, 2000.

[13] S. Kanas, "Coefficient estimates in subclasses of the Caratheodory class related to conical domains," Acta Mathematica Universitatis Comenianae, vol. 74, no. 2, pp. 149-161, 2005.

[14] S. Kanas and D. Răducanu, "Some class of analytic functions related to conic domains," Mathematica Slovaca, vol. 64, no. 5, pp. 1183-1196, 2014.

[15] S. Kanas and S. Altinkaya, "Functions of bounded variation related to domains bounded by conic sections," Mathematica Slovaca, vol. 69, no. 4, pp. 833-842, 2019.

[16] S. Mahmood, M. Arif, and S. N. Malik, "Janowski type closeto-convex functions associated with conic regions," Journal of Inequalities and Applications, vol. 2017, no. 1, p. 259, 2017.

[17] S. Mahmood, M. Jabeen, S. N. Malik, H. M. Srivastava, R. Manzoor, and S. M. Riaz, "Some coefficient inequalities of $q$-starlike functions associated with conic domain defined by q-derivative," Journal of Function Spaces, vol. 2018, Article ID 8492072, 13 pages, 2018.

[18] S. Mahmood, S. N. Malik, S. Farman, S. M. Riaz, and S. Farwa, "Uniformly alpha-quasi-convex functions defined by Janowski functions," Journal of Function Spaces, vol. 2018, Article ID 6049512, 7 pages, 2018.

[19] K. I. Noor and S. N. Malik, "On generalized bounded Mocanu variation associated with conic domain," Mathematical and Computer Modelling, vol. 55, no. 3-4, pp. 844-852, 2012.

[20] M. Naeem, S. Hussain, S. Khan, T. Mahmood, M. Darus, and Z. Shareef, "Janowski type $q$-convex and $q$-close-to-convex functions associated with $q$-conic domain," Mathematics, vol. 8, no. 3, p. 440, 2020.

[21] K. I. Noor, N. Khan, and K. Piejko, "Alpha convex functions associated with conic domains," IJAA, vol. 11, no. 2, pp. 70-80, 2016.

[22] K. I. Noor and S. N. Malik, "On coefficient inequalities of functions associated with conic domains," Computers \& Mathematics with Applications, vol. 62, no. 5, pp. 2209-2217, 2011.

[23] C. Ramachandran, D. Kavitha, and W. Ul-Haq, "Fekete Szegö theorem for a close-to-convex error function," Mathematica Slovaca, vol. 69, no. 2, pp. 391-398, 2019.

[24] C. Ramachandran, L. Vanitha, and S. Kanas, "Certain results on $q$-starlike and $q$-convex error functions," Mathematica Slovaca, vol. 68, no. 2, pp. 361-368, 2018.

[25] S. K. Sahoo and N. L. Sharma, "On a generalization of closeto-convex functions," Annales Polonici Mathematici, vol. 113, no. 1, pp. 93-108, 2015.

[26] H. Shamsan and S. Latha, "On generalized bounded Mocanu variation related to $q$-derivative and conic regions," Annals of Pure and Applied Mathematics, vol. 17, no. 1, pp. 67-83, 2018.

[27] H. M. Srivastava and D. Bansal, "Close-to-convexity of a certain family of q-Mittag-Leffler functions," Journal of Nonlinear and Variational Analysis, vol. 1, no. 1, pp. 61-69, 2017.

[28] H. M. Srivastava, B. Khan, N. Khan, and Q. Z. Ahmad, "Coefficient inequalities for $q$-starlike functions associated with the Janowski functions," Hokkaido Mathematical Journal, vol. 48, no. 2, pp. 407-425, 2019. 